\section{(6) OPEN ACCESS}

\title{
Lipid profile and effect of statin treatment in pooled phase II and phase III baricitinib studies
}

\author{
Peter C Taylor, ${ }^{1}$ Joel M Kremer, ${ }^{2}$ Paul Emery, ${ }^{3}$ Steven H Zuckerman, ${ }^{4}$ Giacomo Ruotolo, ${ }^{4}$ \\ Jinglin Zhong, ${ }^{5}$ Lei Chen, ${ }^{4}$ Sarah Witt, ${ }^{4}$ Chadi Saifan, ${ }^{4}$ Monika Kurzawa, ${ }^{4}$ \\ James D Otvos, ${ }^{6}$ Margery A Connelly, ${ }^{6}$ William L Macias, ${ }^{4}$ Douglas E Schlichting, ${ }^{4}$ \\ Terence P Rooney, ${ }^{4}$ Stephanie de Bono, ${ }^{4}$ Iain B Mclnnes ${ }^{7}$
}

\section{Handling editor Josef $S$ \\ Smolen}

- Additional material is published online only. To view please visit the journal online (http://dx.doi.org/10.1136/ annrheumdis-2017-212461)

For numbered affiliations see end of article.

\section{Correspondence to}

Professor Peter C Taylor, Nuffield Department of Orthopaedics, Rheumatology and Musculoskeletal Sciences, Botnar Research Centre, University of Oxford, Oxford OX3 7LD, UK:

peter.taylor@kennedy.ox.ac.uk

This manuscript is based on work presented at the 2016 ACR/ARHP Annual Meeting: Mclnnes IB, Kremer J, Emery $\mathrm{P}$, Zuckerman $\mathrm{SH}$, Ruotolo G, Saifan C, Chen L, Thanabalasundrum S, Witt $S$ Macias W. Lipid profile and effect of statin treatment in pooled phase 2 and phase 3 baricitinib studies [abstract]. Arthritis Rheumatol. 2016; 68 (suppl 10).

Received 28 September 2017 Revised 2 February 2018 Accepted 3 February 2018 Published Online First 20 February 2018

\section{ABSTRACT}

Objectives Lipid profiles are altered by active disease in patients with rheumatoid arthritis (RA) and may be further modified by treatment with Janus kinase inhibitors and other disease-modifying antirheumatic drugs.

Methods Lipid data were analysed from phase II and III studies of $4 \mathrm{mg}(n=997)$ and $2 \mathrm{mg}(n=479)$ oral baricitinib administered once daily in patients with moderate-to-severe active RA. Lipoprotein particle size and number and GlycA were evaluated with nuclear magnetic resonance in one phase III study. The effect of statin therapy on lipid levels was evaluated in patients on statins at baseline and in patients who initiated statins during the study.

Results Treatment with baricitinib was associated with increased levels of total cholesterol, low-density lipoprotein cholesterol (LDL-C), high-density lipoprotein cholesterol (HDL-C) and triglycerides, but no significant change in LDL-C:HDL-C ratio. Lipid levels plateaued after 12 weeks of treatment. Baricitinib treatment increased large LDL and decreased small, dense LDL particle numbers and GlycA. Lipid changes from baseline were not significantly different between baseline statin users and non-users. In patients who initiated statin therapy during the study, LDL-C, triglycerides (baricitinib $4 \mathrm{mg}$ only) and apolipoprotein $B$ decreased to pre-baricitinib levels; HDL-C and apolipoprotein A-I levels remained elevated.

Conclusions Baricitinib was associated with increased LDL-C, HDL-C and triglyceride levels, but did not alter the LDL-C:HDL-C ratio. Evaluation of cardiovascular event rates during long-term treatment is warranted to further characterise these findings and their possible clinical implications.

Trial registration number NCT00902486, NCT01469013, NCT01185353, NCT01721044, NCT01721057, NCT01711359, NCT01710358, NCT01885078.

\section{INTRODUCTION}

Baricitinib, an oral selective inhibitor of Janus kinase (JAK) 1 and JAK 2, ${ }^{1}$ is approved in the European Union and Japan for the treatment of moderate-to-severe active rheumatoid arthritis (RA) in adults. Baricitinib improved signs and symptoms of RA in phase III, placebo and active-controlled studies in patients with active RA who were naïve to conventional synthetic disease-modifying antirheumatic drugs (csDMARD; RA-BEGIN), ${ }^{2}$ or had an inadequate response (IR) to previous treatment with methotrexate (MTX; RA-BEAM), csDMARDs (RA-BUILD) ${ }^{4}$ and biological DMARDs (RA-BEACON). ${ }^{5}$

In RA, the proinflammatory state in untreated patients is associated with a decrease in total cholesterol, low-density lipoprotein cholesterol (LDL-C) and high-density lipoprotein cholesterol (HDLC) ${ }^{6}$; anti-inflammatory therapies have been shown to increase these lipid levels. ${ }^{7-11}$ Increases in lipids persisted in a phase II study of baricitinib. ${ }^{12}$ The increase in LDL-C was associated with an increase in large and a decrease in small LDL, without any increase in LDL particle number. The increase in HDL-C was associated with an increase in HDL particle number across all particle sizes. ${ }^{12}$ This LDL and HDL particle profile has been associated with reduced atherogenic risk. ${ }^{13}$

This analysis assessed the effects of baricitinib on the lipid profile, lipoprotein particle size and number, and GlycA. GlycA, a measure of glycosylated acute phase proteins, is an emerging inflammatory marker that may be useful for assessing disease activity and is associated with subclinical cardiovascular disease in patients with RA. ${ }^{14-19}$ In addition, the effect of statin therapy on these biomarkers was evaluated. The impact of lipid alteration on cardiovascular risk, changes in risk scores and association between LDL-C change and major adverse cardiovascular events (MACE) were also assessed.

\section{METHODS}

\section{Study design and patients}

Data were included from seven randomised clinical studies: three phase II (JADA, JADC and JADN) and four phase III studies (RA-BEGIN, RA-BEAM, RA-BUILD and RA-BEACON), including data from the long-term extension (LTE) (RA-BEYOND; data through 1 January 2016). All patients completing phase III studies or JADA were eligible to enter RA-BEYOND. The designs for each study have been previously described ${ }^{2-5} 20-23$ and are summarised in online supplementary table S1.

Each study was conducted in accordance with the principles of the Declaration of Helsinki and Good Clinical Practice Guidelines. All patients provided written informed consent. The studies were designed by the sponsors, Eli Lilly and Company and Incyte, 
with input from an academic advisory board in which non-Lilly authors of this manuscript participated. All authors participated in the preparation and review of this manuscript and approved the final version.

\section{Data sets}

Three data sets are defined: the six-study placebo-controlled set, the long-term baricitinib cohort and the all-baricitinib RA-MACE set. The six-study set included all phase II/III studies with placebo and baricitinib $4 \mathrm{mg}$ treatment arms (JADA, JADC and JADN; and RA-BEAM, RA-BUILD and RA-BEACON; JADA, JADN, RA-BUILD and RA-BEACON also had a baricitinib $2 \mathrm{mg}$ treatment arm (online supplementary table S1)). The six-study set included data up to week 24 that were censored at rescue or the end of the placebo-controlled period. The long-term baricitinib cohort comprised patients randomised to baricitinib $4 \mathrm{mg}$ in RA-BEAM, RA-BUILD and RA-BEACON, and patients randomised to baricitinib $2 \mathrm{mg}$ in RA-BUILD and RA-BEACON, including data from RA-BEYOND, with data censored at dose change or rescue in the LTE. The all-baricitinib RA-MACE set included all patients from the phase III studies, including patients in the LTE who received at least one baricitinib dose.

\section{Lipid profile}

Standard lipid panel (total cholesterol, LDL-C, HDL-C and triglycerides) was assessed for all studies; apolipoprotein A-I and apolipoprotein B for JADA and all phase III studies including the LTE; and nuclear magnetic resonance (NMR) spectroscopy panel (lipoprotein particles and GlycA) for JADA and RA-BEAM.

Serum samples for lipid profile were collected in a fasting state at baseline and weeks 12 and 24 after randomisation; results are reported from pooled data of the six-study placebo-controlled set. In the phase III studies, LDL-C was quantified by direct method; in the phase II studies, LDL-C was calculated using the Friedewald equation, unless triglycerides were $>4.52 \mathrm{mmol} / \mathrm{L}$, then LDL-C was quantified by direct method. Lipid treatment-emergent highest values are reported based on criteria from the National Lipids Association Guidelines. ${ }^{24}$ Lipid profile up to 104 weeks was evaluated using the long-term baricitinib cohort.

\section{NMR analysis}

Determination of lipoprotein particle subfractions by NMR was assessed in RA-BEAM, with fasting serum samples collected at baseline and week 12 (LipoScience, now LabCorp, Morrisville, NC). Diameter ranges (nm) were 21.2-23 for large LDL, 18-21.2 for small LDL, $8.8-13$ for large HDL, 8.2-8.8 for medium HDL and 7.3-8.2 for small HDL, as determined by the LP2 algorithm. ${ }^{25}$ Apolipoprotein A-I and apolipoprotein B serum samples were analysed at baseline and weeks 4 and 12 and were quantified using conventional ELISA (Pacific Biomarkers, Seattle, WA). GlycA levels were quantified by NMR in JADA and RA-BEAM at baseline and weeks 12 and 24 as previously described. ${ }^{14}$

\section{Statin use}

Lipid effects by baseline statin use were evaluated in the six-study placebo-controlled set; data are presented for the placebo and baricitinib combined $2 / 4 \mathrm{mg}$ groups. Lipid effects for patients who initiated statins during studies were evaluated in the six-study set for patients randomised to placebo and in the long-term cohort for patients randomised to baricitinib (2 and $4 \mathrm{mg}$ groups presented separately).

\section{Cardiovascular risk assessment}

To evaluate the risk of cardiovascular disease, the Framingham Risk Score ${ }^{26}$ and Reynolds Risk Score ${ }^{2728}$ were assessed at baseline and week 24 in the phase III studies. Relationships were explored in the all-baricitinib RA-MACE set between change in LDL-C and MACE, a composite measure that includes cardiovascular death, stroke and myocardial infarction. An independent, external clinical endpoint committee adjudicated all death and potential cardiovascular events.

\section{Statistical analysis}

Conventional lipid profiles including apolipoprotein A-I and apolipoprotein B were assessed using analysis of covariance (ANCOVA), adjusting for baseline value, study and treatment of analysed lipid measures. The long-term LDL-C and HDL-C profiles were analysed using a restricted maximum likelihood-based mixed model for repeated measures, which included treatment, visit and the treatment-by-visit interaction as fixed categorical effects, and baseline as fixed continuous effect to estimate change from baseline across postbaseline visits after modelling three covariance structures (the heterogeneous autoregressive, compound symmetry and the Toeplitz) and selecting the variance-covariance structure with the smallest Akaike information criterion. ANCOVA was also used to assess the NMR lipoprotein particle parameters and GlycA in RA-BEAM.

For patients who initiated statins during the study, lipid values were evaluated at baseline, initiation of statin therapy and the end of statin treatment or the analysis period. Analysis of variance containing explanatory terms for study and treatment was used. In addition, a subgroup analysis for change from baseline in total cholesterol, LDL-C, HDL-C and triglycerides based on subgroups defined by baseline statin use was performed using an ANCOVA model, with explanatory terms for baseline value, study, treatment, subgroup and treatment-by-subgroup interaction.

The Framingham Risk Score and Reynolds Risk Score were examined from each phase III study using ANCOVA, which included treatment and the baseline measurement as independent variables, and change from baseline as the dependent variable. The association between MACE and LDL-C change was examined using a waterfall plot. The waterfall plot was generated with each individual patient's change of LDL-C from baseline to maximum postbaseline measure, with data censored at treatment discontinuation, or statin therapy initiation or statin therapy change. Changes in LDL-C were ordered from right to left, representing the maximum decrease on the left end and maximum increase on the right end.

\section{RESULTS}

Baseline demographics and disease characteristics of patients from the six-study placebo-controlled set, RA-BEAM and the all-baricitinib RA data were generally comparable among treatment groups (table 1). Vital signs including blood pressure, pulse rate, weight and waist circumference were comparable among treatment groups (data not shown). In the all-baricitinib RA data, $37 \%$ of patients had a medical history of hypertension, $6 \%$ had diabetes at baseline and $31 \%$ were obese $\left(\geq 30 \mathrm{~kg} / \mathrm{m}^{2}\right)$. Previous studies of the phase III trials reported small increases in creatinine with baricitinib treatment. ${ }^{2-5}$

\section{Lipid profile}

In the six-study placebo-controlled set, baricitinib was associated with dose-dependent increases across all lipid 
Table 1 Baseline demographics and disease characteristics in the six-study placebo-controlled set, RA-BEAM, and the all-baricitinib RA set

\begin{tabular}{|c|c|c|c|c|c|c|c|}
\hline & \multicolumn{3}{|c|}{ Six-study placebo-controlled set } & \multicolumn{3}{|l|}{ RA-BEAM } & \multirow[b]{2}{*}{$\begin{array}{l}\text { All-baricitinib RA } \\
(\mathrm{n}=3492)^{*}\end{array}$} \\
\hline & $\begin{array}{l}\text { Placebo } \\
(n=1070)\end{array}$ & $\begin{array}{l}\text { Baricitinib } 4 \mathrm{mg} \\
(\mathrm{n}=997)\end{array}$ & $\begin{array}{l}\text { Baricitinib } 2 \mathrm{mg} \\
(\mathrm{n}=479)\end{array}$ & $\begin{array}{l}\text { Placebo } \\
(n=488)\end{array}$ & $\begin{array}{l}\text { Baricitinib } 4 \mathrm{mg} \\
(\mathrm{n}=487)\end{array}$ & $\begin{array}{l}\text { Adalimumab } \\
(n=330)\end{array}$ & \\
\hline Age, years & $52.9(11.9)$ & $53.7(12.0)$ & $53.2(12.0)$ & $53.4(11.8)$ & $53.5(12.2)$ & $52.9(12.3)$ & $52.9(12.2)$ \\
\hline$\geq 65$ years of age, $n(\%)$ & $173(16)$ & $199(20)$ & $82(17)$ & $82(17)$ & $103(21)$ & $56(17)$ & $612(18)$ \\
\hline $\mathrm{BMI}, \mathrm{kg} / \mathrm{m}^{2}$ & $27.8(7.1)$ & $28.0(6.8)$ & $29.0(7.4)$ & $26.7(6.3)$ & $26.8(5.8)$ & $26.4(5.4)$ & $27.7(6.7)$ \\
\hline Tobacco use, yes, n (\%) & $198(19)$ & $196(20)$ & $84(18)$ & $103(21)$ & $109(22)$ & $74(22)$ & $663(20)$ \\
\hline Female, $n(\%)$ & $862(81)$ & $794(80)$ & $386(81)$ & $382(78)$ & $375(77)$ & $251(76)$ & $2760(79)$ \\
\hline Duration of RAt, years & $8.9(8.4)$ & $8.9(8.6)$ & $9.0(8.1)$ & $8.9(8.0)$ & $8.7(8.6)$ & $8.3(7.9)$ & $7.7(8.2)$ \\
\hline \multicolumn{8}{|l|}{ Region, $\mathrm{n}(\%)$} \\
\hline USA/Canada & $240(22)$ & $225(23)$ & $162(34)$ & $39(8)$ & $40(8)$ & $26(8)$ & $840(24)$ \\
\hline Central/South America and Mexico & $203(19)$ & $197(20)$ & $54(11)$ & $141(29)$ & $143(29)$ & $96(29)$ & $701(20)$ \\
\hline Asia (excluding Japan) & $84(8)$ & $83(8)$ & $38(8)$ & $48(10)$ & $48(10)$ & $33(10)$ & $226(7)$ \\
\hline Japan & $156(15)$ & $132(13)$ & $36(8)$ & $93(191)$ & $93(19)$ & $63(19)$ & $514(15)$ \\
\hline European Union & $263(25)$ & $246(25)$ & $125(26)$ & $116(24)$ & $114(23)$ & $78(24)$ & $783(22)$ \\
\hline Rest of the world & $124(12)$ & $114(11)$ & $64(13)$ & $51(11)$ & $49(10)$ & $34(10)$ & $428(12)$ \\
\hline hsCRP (mg/L), median (first, third quartiles) & $9.9(4.8,24.4)$ & $10.7(5.6,25.4)$ & $9.3(4.9,23.1)$ & $12.7(6.1,26.8)$ & $13.6(6.6,30.4)$ & $14.4(7.2,29.8)$ & $9.1(4.1,22.1)^{*}$ \\
\hline ESR, mm/hour & $46.2(24.9)$ & $46.0(25.1)$ & $43.8(22.3)$ & $49.2(26.1)$ & $49.3(25.8)$ & $48.4(25.6)$ & $42.4(25.4)^{*}$ \\
\hline DAS28-ESR & $6.34(0.99)$ & $6.38(0.96)$ & $6.40(0.99)$ & $6.4(1.0)$ & $6.5(0.9)$ & $6.4(1.0)$ & $5.7(1.5)^{*}$ \\
\hline DAS28-hsCRP & $5.63(0.95)$ & $5.69(0.94)$ & $5.69(0.96)$ & $5.7(1.0)$ & $5.8(0.9)$ & $5.8(0.9)$ & $5.1(1.5)^{*}$ \\
\hline
\end{tabular}

Data are mean (SD) unless otherwise stated.

${ }^{*}$ The $\mathrm{n}$ for the all-baricitinib RA group for disease activity characteristics is 3439 ; it is smaller than the $\mathrm{n}$ for baseline demographics because efficacy baseline measures are only available for phase II/III studies; efficacy baseline measures were not available for a phase I RA study.

tTime from RA diagnosis.

BMI, body mass index; DAS28, DiseaseActivity Score in 28 joints; ESR, erythrocyte sedimentation rate; hSCRP, high-sensitivity C-reactive protein; RA, rheumatoid arthritis.

measures from baseline to week 12. Levels remained stable across groups from week 12 to 24 . Per cent increases were similar from baseline to both weeks 12 and 24 (figure 1). There was no significant change in the LDL-C:HDL-C ratio from baseline at week 12 (placebo $=-0.02$, baricitinib $2 \mathrm{mg}=-0.03$, baricitinib $4 \mathrm{mg}=-0.02$ ) or week 24 (placebo $=-0.03$, baricitinib $2 \mathrm{mg}=-0.04$, baricitinib 4 $\mathrm{mg}=-0.01)$. After the initial increase from baseline to week
12 in patients receiving baricitinib $4 \mathrm{mg}$, LDL-C and HDL-C remained stable through week 104 (online supplementary figure S1).

Correlation analyses with disease activity measures in the baricitinib $2 \mathrm{mg} / 4 \mathrm{mg}$ analysis set revealed weak correlations between lipid changes and disease activity (online supplementary table S2). In patients treated with baricitinib in the RA-BEAM study, increases in LDL-C and HDL-C were correlated with
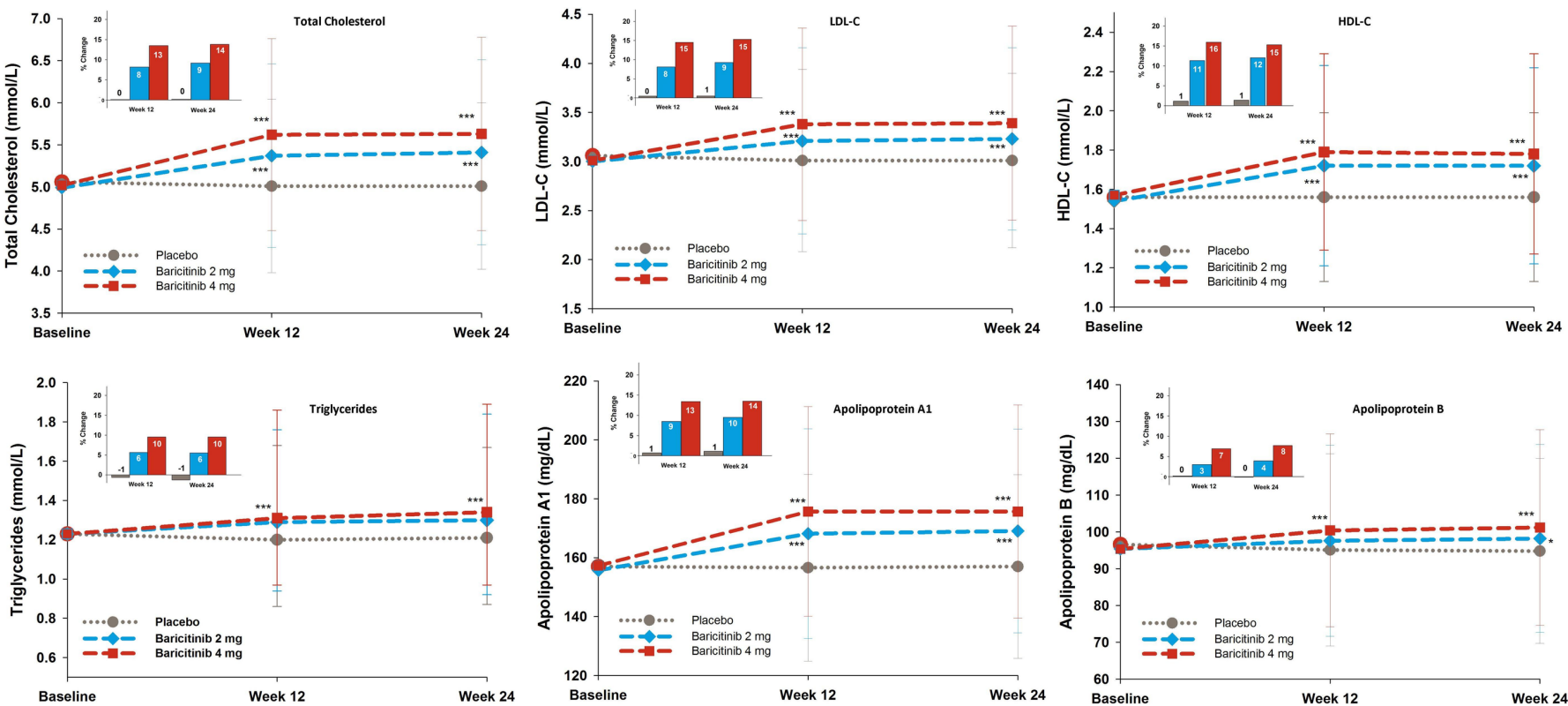

Figure 1 Lipid profile for total cholesterol, LDL-C, HDL-C, triglycerides, apolipoprotein A-I and apolipoprotein B in patients from the six-study placebo-controlled set. Data in line graphs are absolute values at baseline, week 12 and week 24 with data censored at rescue; mean (SD) for all lipids except triglycerides, which are median (25th, 75th percentiles). Patients on placebo who were rescued to baricitinib before week 24 were not included in this analysis. Inset bar charts show mean per cent change from baseline to weeks 12 and 24 with data censored at rescue. * $P \leq 0.05$, ${ }^{* *} \mathrm{P} \leq 0.001$ versus placebo. HDL-C, high-density lipoprotein cholesterol; LDL-C, low-density lipoprotein cholesterol. 
reductions in high-sensitivity C-reactive protein (hsCRP) and GlycA (online supplementary figure S2).

\section{Lipid treatment-emergent highest values}

Baseline abnormal LDL-C values (high or very high) were $11.8 \%$ in the placebo, $10.5 \%$ in the baricitinib $4 \mathrm{mg}$ group and $8.7 \%$ in the baricitinib $2 \mathrm{mg}$ group. Among patients in the baricitinib $4 \mathrm{mg}$ group with a treatment-emergent increase in LDL-C, $92 \%$ of patients did not reach the maximum LDL-C category of $\geq 4.91 \mathrm{mmol} / \mathrm{L}$, and $99 \%$ did not reach the maximum triglyceride category of $\geq 5.64 \mathrm{mmol} / \mathrm{L}$ (online supplementary table S3).

\section{Changes in the NMR lipoprotein profile and GlycA}

In RA-BEAM, there was a significant increase from baseline to week 12 in mean LDL-C in the baricitinib $4 \mathrm{mg}$ group $(0.42 \mathrm{mmol} / \mathrm{L})$ and adalimumab group $(0.20 \mathrm{mmol} / \mathrm{L})$ compared with placebo $(-0.06 \mathrm{mmol} / \mathrm{L}, \mathrm{P}=0.001)$ and in mean HDL-C (baricitinib $4 \mathrm{mg}, \quad 0.24 \mathrm{mmol} / \mathrm{L}$; adalimumab $0.10 \mathrm{mmol} / \mathrm{L}$; placebo $0.00 \mathrm{mmol} / \mathrm{L} ; \quad \mathrm{P}=0.001)$. Statistically significant increases in large LDL particle numbers and decreases in small LDL particle numbers were observed in baricitinib and adalimumab arms compared with placebo (table 2). There was a statistically significant increase in total LDL particle numbers in baricitinib compared with placebo. The increases in large LDL

Table 2 NMR lipoprotein profile: particle subfractions and GlycA in RA-BEAM

\begin{tabular}{|c|c|c|c|}
\hline & $\begin{array}{l}\text { Placebo } \\
\mathrm{n}=488\end{array}$ & $\begin{array}{l}\text { Baricitinib } 4 \mathrm{mg} \\
\mathrm{n}=487\end{array}$ & $\begin{array}{l}\text { Adalimumab } \\
\mathrm{n}=330\end{array}$ \\
\hline \multicolumn{4}{|l|}{ Mean number of particles ( $\mathrm{nmol} / \mathrm{L}$ ) } \\
\hline \multicolumn{4}{|l|}{ Total LDL } \\
\hline Baseline & $1178.60(398.50)$ & $1178.16(335.46)$ & $1165.15(330.16)$ \\
\hline Change from baseline at week 12 & $-21.31(12.25)$ & $15.77(12.09)^{*}++$ & $-36.64(14.77)$ \\
\hline \multicolumn{4}{|l|}{ Large LDL } \\
\hline Baseline & $513.31(216.87)$ & $506.54(225.29)$ & $501.96(227.72)$ \\
\hline Change from baseline at week 12 & $-0.72(8.20)$ & $81.74(8.09)^{* * *}+++$ & $39.49(9.89)^{* *}$ \\
\hline \multicolumn{4}{|l|}{ Small LDL } \\
\hline Baseline & $632.45(415.25)$ & $637.68(374.39)$ & $633.34(372.48)$ \\
\hline Change from baseline at week 12 & $-21.86(15.09)$ & $-80.26(14.89)^{* *}$ & $-82.86(18.18)^{* *}$ \\
\hline \multicolumn{4}{|l|}{ Medium small LDL } \\
\hline Baseline & $127.25(83.21)$ & $130.05(77.97)$ & $128.35(77.36)$ \\
\hline Change from baseline at week 12 & $-3.18(3.15)$ & $-16.29(3.11)^{* *}$ & $-15.47(3.80)^{*}$ \\
\hline \multicolumn{4}{|l|}{ Very small LDL } \\
\hline Baseline & $505.26(333.70)$ & $507.64(298.81)$ & $504.94(297.13)$ \\
\hline Change from baseline at week 12 & $-18.76(12.07)$ & $-63.93(11.91)^{\star *}$ & $-67.37(14.55)^{*}$ \\
\hline \multicolumn{4}{|l|}{ Total HDL } \\
\hline Baseline & $28.88(5.90)$ & $28.69(5.96)$ & $28.48(5.84)$ \\
\hline Change from baseline at week 12 & $0.06(0.22)$ & $4.73(0.21)^{* * *}+++$ & $1.93(0.26)^{* * *}$ \\
\hline \multicolumn{4}{|l|}{ Large HDL } \\
\hline Baseline & $9.01(3.41)$ & $9.13(3.48)$ & $8.91(3.56)$ \\
\hline Change from baseline at week 12 & $0.00(0.12)$ & $0.98(0.12)^{* * *}++$ & $0.41(0.14)^{*}$ \\
\hline \multicolumn{4}{|l|}{ Medium HDL } \\
\hline Baseline & $2.82(3.27)$ & $2.68(3.01)$ & $2.89(3.27)$ \\
\hline Change from baseline at week 12 & $0.13(0.14)$ & $0.63(0.14)^{*}+++$ & $-0.30(0.17)^{*}$ \\
\hline \multicolumn{4}{|l|}{ Small HDL (nmol/L) } \\
\hline Baseline & $17.04(5.59)$ & $16.88(5.66)$ & $16.68(5.54)$ \\
\hline Change from baseline at week 12 & $-0.08(0.21)$ & $3.08(0.21)^{* * *}+++$ & $1.86(0.25)^{* * *}$ \\
\hline \multicolumn{4}{|l|}{ Mean particle size (nm) } \\
\hline \multicolumn{4}{|l|}{ LDL particle size } \\
\hline Baseline & $21.34(0.76)$ & $21.29(0.75)$ & $21.28(0.74)$ \\
\hline Change from baseline at week 12 & $0.02(0.03)$ & $0.25(0.03)^{* * *}$ & $0.23(0.03)^{* * *}$ \\
\hline \multicolumn{4}{|l|}{ VLDL particle size } \\
\hline Baseline & $47.59(9.29)$ & $46.94(9.09)$ & $47.90(11.61)$ \\
\hline Change from baseline at week 12 & $0.57(0.40)$ & $2.34(0.40)^{* *}$ & $1.19(0.49)$ \\
\hline \multicolumn{4}{|l|}{ HDL particle size } \\
\hline Baseline & $9.34(0.47)$ & $9.33(0.46)$ & $9.36(0.49)$ \\
\hline Change from baseline at week 12 & $0.01(0.01)$ & $-0.02(0.01)^{*}$ & $-0.00(0.01)$ \\
\hline \multicolumn{4}{|l|}{ Mean levels of GlycA ( $\mu \mathrm{mol} / \mathrm{L})$} \\
\hline Baseline & $508.9(104.6)$ & $517.2(114.4)$ & $513.9(106.4)$ \\
\hline Change from baseline at week 12 & $-13.6(3.8)$ & $-110.9(3.8)^{* * *}+$ & $-98.7(4.7)^{* * *}$ \\
\hline
\end{tabular}

Baseline data are mean (SD); change from baseline data are least squares mean (SE).

${ }^{*} P \leq 0.05 ;{ }^{* *} P \leq 0.01 ;{ }^{* * *} P \leq 0.001$ versus placebo. $+P \leq 0.05 ;++P \leq 0.01 ;+++P \leq 0.001$ versus adalimumab.

HDL, high-density lipoprotein; LDL, low-density lipoprotein; NMR, nuclear magnetic resonance; RA, rheumatoid arthritis; VLDL, very-low-density lipoprotein. 
Table 3 Change from baseline in lipids by baseline statin use in phase III studies up to 24 weeks with data censored at rescue

\begin{tabular}{|c|c|c|c|c|c|}
\hline & \multicolumn{2}{|l|}{ Placebo } & \multicolumn{2}{|l|}{ Baricitinib 2/4 mg } & \multirow[b]{2}{*}{ Interaction $\mathrm{P}$ value } \\
\hline & Non-statin $(n=704)$ & Statin $(n=84)$ & Non-statin $(n=1054)$ & Statin $(n=137)$ & \\
\hline \multicolumn{6}{|l|}{ Total cholesterol, mmol/L } \\
\hline Baseline & $5.03(1.00)$ & $5.13(1.20)$ & $5.01(1.01)$ & $4.88(1.05)$ & \\
\hline Change from baseline to week 24 & $-0.01(0.03)$ & $0.17(0.11)$ & $0.59(0.02)$ & $0.63(0.08)$ & 0.505 \\
\hline \multicolumn{6}{|l|}{ LDL-C, mmol/L } \\
\hline Baseline & $3.04(0.86)$ & $3.00(1.08)$ & $3.03(0.84)$ & $2.81(0.85)$ & \\
\hline Change from baseline to week 24 & $-0.03(0.02)$ & $0.20(0.10)$ & $0.35(0.02)$ & $0.41(0.07)$ & 0.110 \\
\hline \multicolumn{6}{|l|}{$\mathrm{HDL}-\mathrm{C}, \mathrm{mmol} / \mathrm{L}$} \\
\hline Baseline & $1.56(0.42)$ & $1.47(0.40)$ & $1.57(0.42)$ & $1.48(0.36)$ & \\
\hline Change from baseline to week 24 & $-0.00(0.01)$ & $0.00(0.03)$ & $0.20(0.01)$ & $0.24(0.02)$ & 0.454 \\
\hline \multicolumn{6}{|l|}{ Triglycerides, mmol/L } \\
\hline Baseline & $1.38(0.71)$ & $1.93(1.83)$ & $1.38(0.74)$ & $1.72(0.96)$ & \\
\hline Change from baseline to week 24 & $-0.01(0.03)$ & $-0.01(0.12)$ & $0.15(0.02)$ & $0.14(0.09)$ & 0.730 \\
\hline
\end{tabular}

Baseline are mean (SD).

Change from baseline data are least squares mean (SE).

HDL-C, high-density lipoprotein cholesterol; LDL-C, low-density lipoprotein cholesterol.

particles and decreases in the smaller LDL particles produced an overall mean increase at 12 weeks in LDL size for baricitinib $(0.25 \mathrm{~nm})$ and adalimumab $(0.23 \mathrm{~nm})$ that was significantly greater than placebo $(0.02 \mathrm{~nm} ; \mathrm{P}=0.001)$ (table 2$)$.

Total HDL particle numbers increased with statistically significant elevations in large, medium and small HDL particles for baricitinib and in large and small HDL particles for adalimumab compared with placebo (table 2). The overall impact on mean HDL particle size for baricitinib was a modest decrease at week $12(-0.02 \mathrm{~nm})$, which was not observed for adalimumab $(0.00 \mathrm{~nm})$. A statistically significant within-group increase from baseline in the mean very-low-density lipoprotein particle size was observed in baricitinib $(2.34 \mathrm{nmol} / \mathrm{L})$ and adalimumab $(1.19 \mathrm{nmol} / \mathrm{L})$; the increase in baricitinib $4 \mathrm{mg}$ was statistically significant compared with placebo $(0.57 \mathrm{nmol} / \mathrm{L} ; \mathrm{P}=0.002)$ (table 2). There were dose-dependent decreases in GlycA levels from baseline to week 12 in JADA $(-47.30,-49.80,-58.46$ and $-80.55 \mu \mathrm{mol} / \mathrm{L}$ in the baricitinib $1,2,4$ and $8 \mathrm{mg}$ dose groups, respectively). In RA-BEAM at week 12, GlycA levels in patients treated with baricitinib decreased significantly compared with placebo or adalimumab (table 2). There were similar reductions in GlycA with baricitinib regardless of baseline statin use (online supplementary table S4).

\section{Statin use}

Approximately $10 \%(n=221)$ of patients were on statin therapy at baseline. In the baricitinib $2 / 4 \mathrm{mg}$ group, total cholesterol, LDL-C, HDL-C and triglycerides increased from baseline in both statin users and non-users (table 3 ). In the placebo group, total cholesterol, LDL-C, HDL-C and triglycerides remained essentially unchanged in non-statin users, while there was a modest increase from baseline in total cholesterol and LDL-C in statin users without much of a change in HDL-C or triglycerides (table 3). The interaction effect between study treatment and baseline statin subgroup was non-significant $(P>0.1)$, suggesting that baseline statin use did not modify the effect of baricitinib on lipids.

Twenty patients in the placebo group, and 58 patients in the baricitinib $4 \mathrm{mg}$ and 25 patients in the baricitinib $2 \mathrm{mg}$ long-term cohorts initiated statin therapy after starting baricitinib and had lipid data available at baseline, statin initiation and the end of statin treatment. Figure 2 shows changes in lipid levels for these patients at each of these time points. The effects of statin therapy on LDL-C, total cholesterol, triglycerides and apolipoprotein B lipid levels were comparable in the baricitinib and placebo groups.

\section{Cardiovascular risk scores and MACE}

By baseline Framingham Risk Score, the majority of patients aged 30-74 years with no prior cardiovascular disease had low or intermediate risk in the phase III studies in each treatment group; less than $12 \%$ were considered high risk. There were no statistically significant within-group changes from baseline or statistically significant differences between groups (baricitinib $4 \mathrm{mg}$, placebo or adalimumab) (table 4). For the Reynolds Risk Score, most non-diabetic patients aged 45-80 with no prior cardiovascular disease (any hsCRP status and the subgroup with baseline hsCRP $\leq 20 \mathrm{mg} / \mathrm{L}$ ) had baseline scores of low or low-to-moderate risk in the phase III studies. Less than $13 \%$ of patients were considered moderate to high or high risk. There was a statistically significant within-group decrease in Reynolds Risk Score for baricitinib groups in all three studies and for adalimumab in RA-BEAM as well as placebo in RA-BUILD. Only in RA-BEAM were the decreases significantly larger in the baricitinib group compared with placebo (table 4).

In the all-baricitinib RA-MACE set, 25 patients had positively adjudicated MACE (incidence rate $=0.57$ per 100 patient-years; patient-years of exposure $=4402$ ). There was no observed association between change in LDL-C and occurrence of MACE (online supplementary figure S3).

\section{DISCUSSION}

RA confers increased risk of cardiovascular events that are not explained fully by factors that contribute to cardiovascular risk in the general population. This has led to the suggestion that inflammation mediates direct effects on cardiovascular risk in RA and that interventions that modulate inflammatory burden may favourably influence the risk of a vascular event. Specifically, MTX is associated with reduced cardiovascular mortality in RA. ${ }^{29}$ Tumour necrosis factor (TNF) inhibitors are similarly associated with reduced vascular mortality and event rates. ${ }^{30}$

Referred to as the RA lipid paradox, active disease is associated with 'lower than expected' cholesterol levels that in turn 

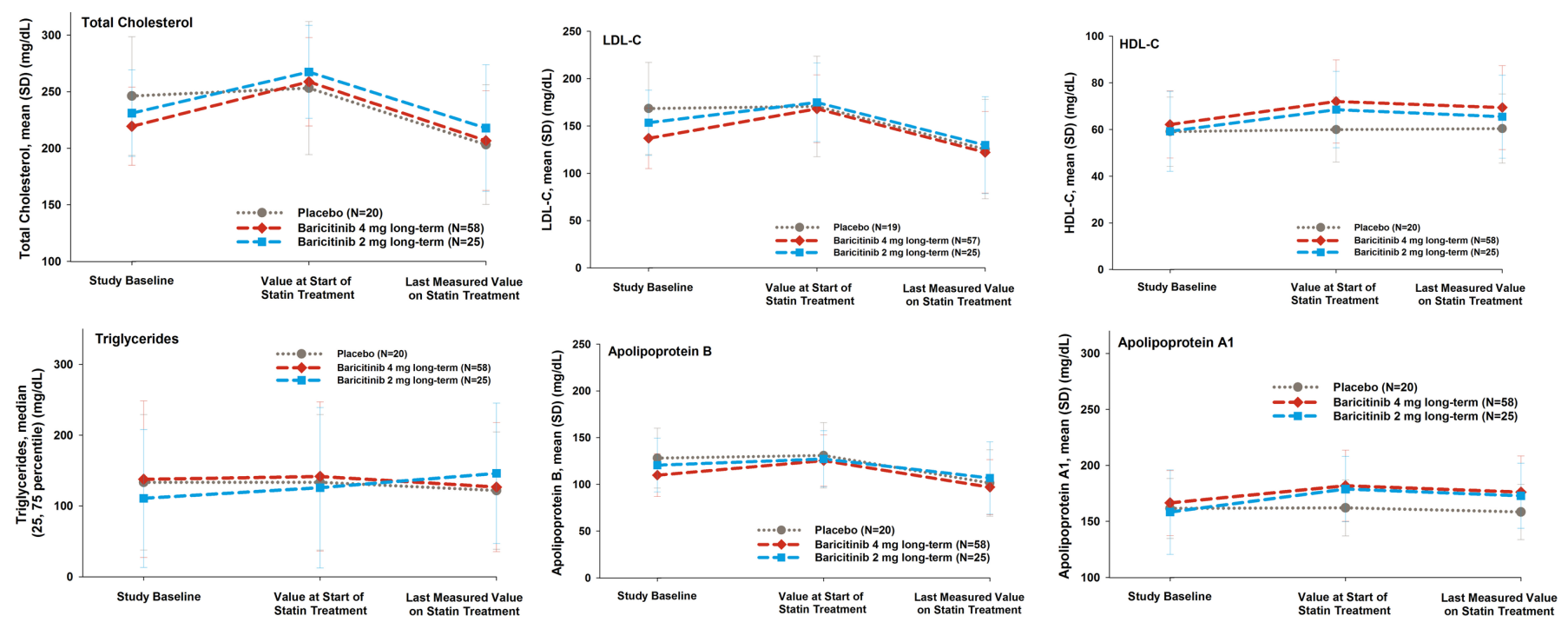

Figure 2 Lipid profile in patients who initiated statins during the phase III studies of the six-study placebo-controlled set including data in the longterm RA-BEYOND study for the baricitinib doses. Data are absolute values at three milestones: baseline, initiation of statin therapy and end of statin treatment or study period; mean (SD) for all lipids except triglycerides, which are median (25th, 75th percentiles). HDL-C, high-density lipoprotein cholesterol; LDL-C, low-density lipoprotein cholesterol.

may 'normalize' on effective management of the inflammatory disease state ${ }^{31}{ }^{32}$ having several implications. First, it is difficult to determine the long-term impact of lipid changes in the context of treatment of active inflammation as in RA. For example, some effects of baricitinib, especially given the behaviour of the adalimumab control arm, likely do reflect the impact of normalisation of cholesterol levels. However, like tocilizumab and tofacitinib, the magnitude of change seems greater and more consistent for baricitinib than for TNF inhibitors and csDMARDs in historical comparator studies, and is likely to be target specific. However, in a recent clinical trial analysing MACE in patients with RA, no differences in event rates were observed when comparing tocilizumab with etanercept. ${ }^{33}$ In our analysis, in general, we found low MACE rates with no increase over time and no apparent association between LDL-C change and MACE at the individual patient level. These data are not to be overinterpreted, but they do reassure that no immediate clinical effect is manifest on lipid changes in an at-risk population-about $12 \%$ of patients entering these studies were already in a high-risk category on recruitment. In this respect, long-term observational data will be important across the field and distinct mechanisms of action.
Overall trends in baricitinib NMR results observed previously were confirmed in this analysis of phase III data from RA-BEAM, with a few exceptions. In the phase II JADA study, there was a trend towards significance for decreases of small, medium small and very small LDL particles at 12 weeks $^{12}$; in RA-BEAM these differences were all significant. Also, in JADA there was no increase in total LDL particle number; in RA-BEAM there was a small, but significant, increase in total LDL particle number in baricitinib versus placebo and adalimumab.

One possible explanation for the mechanism by which baricitinib and related interventions increase LDL particle size could be the increased activity of lipases such as phospholipase A2, hepatic lipase, lipoprotein lipase and endothelial lipase, which are reported to be increased in chronic inflammatory states. ${ }^{34-37}$ Lipase levels were not investigated in this analysis, but it is possible that some lipases, specifically sPLA2, an acute phase reactant, could be reduced by an overall baricitinib-mediated reduction in systemic inflammation and thereby reduce the rate of lipoprotein remodelling. A reduction in inflammation-stimulated lipase activity could then contribute to a shift in the equilibrium towards larger lipoprotein particles. This

Table 4 Change in cardiovascular risk scores from baseline to week 24 with data censored at rescue

\begin{tabular}{|c|c|c|c|c|c|c|c|c|c|}
\hline & \multicolumn{3}{|l|}{ RA-BEAM } & \multicolumn{3}{|l|}{ RA-BUILD } & \multicolumn{3}{|l|}{ RA-BEACON } \\
\hline & $\begin{array}{l}\text { Placebo } \\
(n=488)\end{array}$ & $\begin{array}{l}\text { Baricitinib } \\
4 \mathrm{mg}(\mathrm{n}=487)\end{array}$ & $\begin{array}{l}\text { Adalimumab } \\
(\mathrm{n}=330)\end{array}$ & $\begin{array}{l}\text { Placebo } \\
(n=228)\end{array}$ & $\begin{array}{l}\text { Baricitinib } \\
2 \mathrm{mg}(\mathrm{n}=229)\end{array}$ & $\begin{array}{l}\text { Baricitinib } \\
4 \mathrm{mg}(\mathrm{n}=227)\end{array}$ & $\begin{array}{l}\text { Placebo } \\
(n=126)\end{array}$ & $\begin{array}{l}\text { Baricitinib } \\
2 \mathrm{mg}(\mathrm{n}=127)\end{array}$ & $\begin{array}{l}\text { Baricitinib } 4 \mathrm{mg} \\
(\mathrm{n}=137)\end{array}$ \\
\hline \multicolumn{10}{|l|}{ Framingham Risk Score } \\
\hline Baseline & $9.9(9.9)$ & $8.8(9.6)$ & $9.6(10.0)$ & $7.7(6.8)$ & $7.6(6.4)$ & $7.5(6.6)$ & $8.5(6.3)$ & $8.0(6.4)$ & $8.2(8.0)$ \\
\hline $\begin{array}{l}\text { Change from baseline to } \\
\text { week } 24\end{array}$ & $0.02(0.19)$ & $0.28(0.17)$ & $-0.07(0.21)$ & $-0.30(0.25)$ & $0.02(0.22)$ & $-0.01(0.22)$ & $-0.24(0.39)$ & $-0.46(0.34)$ & $0.63(0.32)$ \\
\hline \multicolumn{10}{|l|}{ Reynolds Risk Score } \\
\hline Baseline & $5.4(6.7)$ & $4.5(4.6)$ & $5.3(6.1)$ & $4.7(5.0)$ & $3.9(3.6)$ & $4.4(4.2)$ & $5.1(5.8)$ & $4.3(4.5)$ & $4.6(4.1)$ \\
\hline $\begin{array}{l}\text { Change from baseline to } \\
\text { week } 24\end{array}$ & $-0.11(0.16)$ & $-0.98(0.15)^{*} \dagger$ & $-1.08(0.18)^{*} \dagger$ & $-0.59(0.20) \ddagger$ & $-0.66(0.17)^{*}$ & $-0.47(0.17) \dagger$ & $-0.32(0.29)$ & $-0.74(0.26) \ddagger$ & $-0.69(0.26) \ddagger$ \\
\hline
\end{tabular}

Baseline are mean (SD).

Change from baseline data are least squares mean (SE).

* Significant within-group change from baseline $(\mathrm{P}<0.001)$.

tSignificant change from baseline versus placebo $(P<0.001)$.

¥Significant within-group change from baseline $(P<0.01)$.

$R A$, rheumatoid arthritis. 
shift in larger lipoprotein particles could reflect a reduction in the atherogenic potential of the overall LDL particles.

GlycA, a measure of glycosylated acute phase proteins, is an emerging inflammatory marker that may be useful for assessing disease activity and is associated with subclinical cardiovascular disease in patients with RA. ${ }^{15}{ }^{19}$ Baricitinib decreased GlycA dose dependently and reductions were seen regardless of baseline statin treatment. With recent studies suggesting GlycA as a marker for cardiovascular risk, these reductions in phase II and III studies in an RA population may portend a reduction in overall cardiovascular risk.

While in JADA there was no increase in LDL-C levels in patients on statins at baseline, ${ }^{12}$ the current analysis of pooled baricitinib data showed that about $10 \%$ of patients already receiving statins on study entry demonstrated increases in lipids. It is not known if increasing statin dose or changing to a more potent statin would reverse this effect. Meanwhile, for patients starting a statin during the study, total cholesterol and LDL-C returned to baseline levels, whereas HDL-C remained elevated. Consistent with these findings, reduction in elevated cholesterol secondary to tofacitinib has previously been reported following initiation of atorvastatin. ${ }^{38}$

This study has some limitations. First, the observation period for the placebo-controlled comparisons is short (24 weeks) due to the need to limit the duration of placebo treatment. Therefore, long-term effects of baricitinib on lipid levels are based on within-group comparisons only. Second, the effects of baseline statin use on baricitinib-induced lipid changes did not account for postbaseline changes in statin dose or change to a different statin. Also, because a limited number of patients initiated statin therapy during the placebo-controlled period, we extended the analysis for statin effect to include data from the LTE for patients receiving baricitinib 2 and $4 \mathrm{mg}$ treatment, while placebo data are based on 0-24 weeks; therefore, these effects are based on a non-randomised comparison of patients starting statin treatment. As a result, caution is warranted when interpreting the number of patients initiating statin as a proxy for the number of patients who need statin therapy. Third, while the majority of these analyses are from pooled data from multiple baricitinib studies, the NMR data were only collected and assessed in one phase III study in MTX-IR patients (RA-BEAM) and therefore we could not present these results from the broader patient population across the baricitinib programme. We used the Framingham Risk Score and Reynolds Risk Score to characterise the change of cardiovascular risk, which may not reflect well the cardiovascular risk in our target population given the lipid paradox in patients with RA. Additionally, the duration of follow-up for cardiovascular events in our analysis is limited to 24 weeks in the placebo-controlled portion of the studies and limited by the data cut-off date in the LTE. We continue to monitor cardiovascular events in extension studies.

In summary, increases in circulating lipid levels were seen following treatment with baricitinib that plateaued by 12 weeks of treatment. Increases in HDL occurred across all particle sizes whereas LDL increases were confined to larger particles, with small LDL particle levels decreasing. LDL increases reversed in response to statin therapy. These findings may reflect a positive impact on aspects of the lipid paradox observed in RA. However, further analyses of the effects of baricitinib on cholesterol and lipoprotein metabolism, as well as continued evaluation of cardiovascular event rates during long-term treatment, are warranted to further characterise these findings and their possible clinical implications.

\section{Author affiliations}

${ }^{1}$ Botnar Research Centre, Nuffield Department ofOrthopaedics, Rheumatology and Musculoskeletal Sciences, University of Oxford, Oxford, UK

${ }^{2}$ Center for Rheumatology, Albany Medical College, Albany, New York, USA

${ }^{3}$ Leeds Institute of Rheumatic and Musculoskeletal Medicine, University of Leeds, Leeds, UK

${ }^{4}$ Eli Lilly and Company, Indianapolis, Indiana, USA

${ }^{5}$ OVVIA, Morrisville, North Carolina, USA

${ }^{6}$ Laboratory Corporation of America Holdings (LabCorp), Morrisville, North Carolina, USA

${ }^{7}$ Institute of Infection, Immunity and Inflammation, College of Medical, Veterinary and Life Sciences, University of Glasgow, Glasgow, UK

Acknowledgements The authors thank Kathy Oneacre, MA, of Syneos Health, funded by Eli Lilly and Company, for medical writing support and assistance with preparation and submission of this manuscript. PCT would like to acknowledge the support of the National Institute for Health Research (NIHR) Oxford Biomedical Research Centre (BRC).

Contributors PCT participated in the conception and design of the study, interpreted the data, drafted the manuscript and provided critical revision for important intellectual content. JMK participated in the design of the work, acquisition, analysis and interpretation of data for the work, and drafted and provided critical revision of the manuscript for important intellectual content. PE participated in the design of the work and interpretation of data for the work, and drafted and provided critical revision of the manuscript for important intellectual content. SHZ participated in the conception of the work, analysis and interpretation of data for the work, and drafted and provided critical revision of the manuscript for important intellectual content. GR and MK participated in the interpretation of data for the work, and provided critical revision of the manuscript for important intellectual content. JZ, JDO, MAC and TPR participated in the acquisition, analysis and interpretation of data for the work, and provided critical revision of the manuscript for important intellectual content. LC participated in the conception and design of the work, analysis and interpretation of data for the work, and provided critical revision of the manuscript for important intellectual content. SW participated in acquisition and analysis of data for the study and provided critical revision for important intellectual content. CS participated in the analysis and interpretation of data for the work, and provided critical revision of the manuscript for important intellectual content. WLM participated in analysis of data for the work, and provided critical revision of the manuscript for important intellectual content. DES and IBM participated in the interpretation of data for the work, and drafted and provided critical revision of the manuscript for important intellectual content. SdB participated in the acquisition and interpretation of data for the work, and provided critical revision of the manuscript for important intellectual content. All authors provided critical input and approval to the final manuscript.

Funding This study was funded by Eli Lilly and Company and Incyte.

Disclaimer The views expressed are those of the author and not necessarily those of the National Health Service, the NIHR or the Department of Health.

Competing interests PCT reports personal fees from Eli Lilly and Company during the conduct of the study; grant support from Celgene and Galapagos, grant support and personal fees from UCB Pharma, and personal fees from AbbVie, Bristol-Myers Squibb, GlaxoSmithKline, Merck, Pfizer and Takeda outside the submitted work. JMK reports grant support and personal fees from Eli Lilly and Company during the conduct of the study; grant support from Pfizer and Novartis, grant support and personal fees from Eli Lilly and Company; and other support from Corrona outside the submitted work. PE reports grant support and personal fees from Eli Lilly and Company during the conduct of this study and grant support from Pfizer, MSD, AbbVie, BMS, UCB, Roche, Novartis, Samsung and Sandoz. SHZ, GR, LC, SW, CS, MK, WLM, DES, TPR and SdB are full-time employees and stockholders of Eli Lilly and Company. JZ is employed by IQVIA with which Eli Lilly and Company contracts for clinical trial and statistical support. JDO reports employment by LabCorp during the conduct of the study. In addition, JDO is an inventor on an issued patent: NMR measurements of GlycA. MAC is an employee of LabCorp. IBM reports grant support and personal fees from Eli Lilly and Company during the conduct of this study and grant support from AbbVie, Pfizer, Novartis, Roche, Janssen and Pfizer.

Patient consent Obtained.

Ethics approval The studies were approved by each center's Institutional Review Board or Ethics Committee.

Provenance and peer review Not commissioned; externally peer reviewed. Data sharing statement There are no additional data available.

Open access This is an Open Access article distributed in accordance with the Creative Commons Attribution Non Commercial (CC BY-NC 4.0) license, which permits others to distribute, remix, adapt, build upon this work non-commercially, and license their derivative works on different terms, provided the original work 
is properly cited and the use is non-commercial. See: http://creativecommons.org/ licenses/by-nc/4.0/

(c) Article author(s) (or their employer(s) unless otherwise stated in the text of the article) 2018. All rights reserved. No commercial use is permitted unless otherwise expressly granted.

\section{REFERENCES}

1 Fridman JS, Scherle PA, Collins R, et al. Selective inhibition of JAK1 and JAK2 is efficacious in rodent models of arthritis: preclinical characterization of INCB028050. $J$ Immunol 2010;184:5298-307.

2 Fleischmann R, Schiff M, van der Heijde D, et al. Baricitinib, Methotrexate, or Combination in Patients With Rheumatoid Arthritis and No or Limited Prior DiseaseModifying Antirheumatic Drug Treatment. Arthritis Rheumatol 2017;69:506-17.

3 Taylor PC, Keystone EC, van der Heijde D, et al. Baricitinib versus placebo or adalimumab in rheumatoid arthritis. N Engl J Med 2017;376:652-62.

4 Dougados M, van der Heijde D, Chen YC, et al. Baricitinib in patients with inadequate response or intolerance to conventional synthetic DMARDs: results from the RA-BUILD study. Ann Rheum Dis 2017;76:88-95.

5 Genovese MC, Kremer J, Zamani 0, et al. Baricitinib in Patients with Refractory Rheumatoid Arthritis. N Engl J Med 2016;374:1243-52.

6 Robertson J, Peters MJ, McInnes IB, et al. Changes in lipid levels with inflammation and therapy in RA: a maturing paradigm. Nat Rev Rheumatol 2013;9:513-23.

7 O'Neill F, Charakida M, Topham E, et al. Anti-inflammatory treatment improves highdensity lipoprotein function in rheumatoid arthritis. Heart 2017;103:766-73.

8 Tam LS, Tomlinson B, Chu TT, et al. Impact of TNF inhibition on insulin resistance and lipids levels in patients with rheumatoid arthritis. Clin Rheumatol 2007;26:1495-8.

9 Navarro-Millán I, Charles-Schoeman C, Yang S, et al. Changes in lipoproteins associated with methotrexate or combination therapy in early rheumatoid arthritis: results from the treatment of early rheumatoid arthritis trial. Arthritis Rheum 2013;65:1430-8.

10 Genovese MC, McKay JD, Nasonov EL, et al. Interleukin-6 receptor inhibition with tocilizumab reduces disease activity in rheumatoid arthritis with inadequate response to disease-modifying antirheumatic drugs: the tocilizumab in combination with traditional disease-modifying antirheumatic drug therapy study. Arthritis Rheum 2008; 58:2968-80

11 Charles-Schoeman C, Fleischmann R, Davignon J, et al. Potential mechanisms leading to the abnormal lipid profile in patients with rheumatoid arthritis versus healthy volunteers and reversal by tofacitinib. Arthritis Rheumatol 2015:67:616-25.

12 Kremer JM, Genovese MC, Keystone E, et al. Effects of Baricitinib on Lipid, Apolipoprotein, and Lipoprotein Particle Profiles in a Phase Ilb Study of Patients With Active Rheumatoid Arthritis. Arthritis Rheumatol 2017;69:943-52.

13 Rizzo M, Berneis K. Low-density lipoprotein size and cardiovascular risk assessment. QJM 2006;99:1-14.

14 Otvos JD, Shalaurova I, Wolak-Dinsmore J, et al. GlycA: a composite nuclear magnetic resonance biomarker of systemic inflammation. Clin Chem 2015;61:714-23.

15 Ormseth MJ, Chung CP, Oeser AM, et al. Utility of a novel inflammatory marker, GlycA, for assessment of rheumatoid arthritis disease activity and coronary atherosclerosis. Arthritis Res Ther 2015;17:117.

16 Bartlett DB, Connelly MA, AbouAssi H, et al. A novel inflammatory biomarker, GlycA, associates with disease activity in rheumatoid arthritis and cardio-metabolic risk in BMI-matched controls. Arthritis Res Ther 2016;18:86.

17 Connelly MA, Gruppen EG, Otvos JD, et al. Inflammatory glycoproteins in cardiometabolic disorders, autoimmune diseases and cancer. Clin Chim Acta 2016:459:177-86.

18 AbouAssi H, Connelly MA, Bateman LA, et al. Does a lack of physical activity explain the rheumatoid arthritis lipid profile? Lipids Health Dis 2017;16:39.
19 Joshi AA, Lerman JB, Aberra TM, et al. GlycA is a novel biomarker of inflammation and subclinical cardiovascular disease in Psoriasis. Circ Res 2016;119:1242-53.

20 Greenwald MW, Fidelus-Gort R, Levy R, et al. A randomized dose-ranging, placebocontrolled study of INCB028050, a selective JAK1 and JAK2 inhibitor in subjects with active rheumatoid arthritis. Arthritis Rheum 2010;62(Suppl 10):2172.

21 Keystone EC, Taylor PC, Drescher E, et al. Safety and efficacy of baricitinib at 24 weeks in patients with rheumatoid arthritis who have had an inadequate response to methotrexate. Ann Rheum Dis 2015:74:333-40.

22 Tanaka Y, Emoto K, Cai Z, et al. Efficacy and Safety of Baricitinib in Japanese Patients with Active Rheumatoid Arthritis Receiving Background Methotrexate Therapy: A 12-week, Double-blind, Randomized Placebo-controlled Study. J Rheumatol 2016:43:504-11.

23 Smolen J, Genovese M, Takeuchi T, et al. THU0166 Safety Profile of Baricitinib in Patients with Active RA: An Integrated Analysis: Table 1. Ann Rheum Dis 2016;75(Suppl 2):243.2-4.

24 Jacobson TA, Ito MK, Maki KC, et al. National Lipid Association recommendations for patient-centered management of dyslipidemia: part 1 - executive summary. J Clin Lipidol 2014;8:473-88.

25 Jeyarajah EJ, Cromwell WC, Otvos JD. Lipoprotein particle analysis by nuclear magnetic resonance spectroscopy. Clin Lab Med 2006;26:847-70.

26 Wilson PW, D'Agostino RB, Levy D, et al. Prediction of coronary heart disease using risk factor categories. Circulation 1998;97:1837-47.

27 Ridker PM, Buring JE, Rifai N, et al. Development and validation of improved algorithms for the assessment of global cardiovascular risk in women: the Reynolds Risk Score. JAMA 2007:297:611-9.

28 Ridker PM, Paynter NP, Rifai N, et al. C-reactive protein and parental history improve global cardiovascular risk prediction: the Reynolds Risk Score for men. Circulation 2008;118:2243-51.

29 Marks JL, Edwards CJ. Protective effect of methotrexate in patients with rheumatoid arthritis and cardiovascular comorbidity. Ther Adv Musculoskelet Dis 2012;4:149-57.

30 Greenberg JD, Kremer JM, Curtis JR, et al. CORRONA Investigators. Tumour necrosis factor antagonist use and associated risk reduction of cardiovascular events among patients with rheumatoid arthritis. Ann Rheum Dis 2011;70:576-82.

31 Myasoedova E, Crowson CS, Kremers HM, et al. Lipid paradox in rheumatoid arthritis: the impact of serum lipid measures and systemic inflammation on the risk of cardiovascular disease. Ann Rheum Dis 2011;70:482-7.

32 Choy E, Ganeshalingam K, Semb AG, et al. Cardiovascular risk in rheumatoid arthritis: recent advances in the understanding of the pivotal role of inflammation, risk predictors and the impact of treatment. Rheumatology 2014;53:2143-54.

33 Giles JT, Sattar N, Gabriel SE, et al. Cardiovascular risk in rheumatoid arthritis: recent advances in the understanding of the pivotal role of inflammation, risk predictors and the impact of treatment. Arthritis Rheumatol 2016;68(Suppl 10):4357-9.

34 Mclnnes IB, Kim HY, Lee SH, et al. Open-label tofacitinib and double-blind atorvastatin in rheumatoid arthritis patients: a randomised study. Ann Rheum Dis 2014:73:124-31.

35 Kitas GD, Gabriel SE. Cardiovascular disease in rheumatoid arthritis: state of the art and future perspectives. Ann Rheum Dis 2011;70:8-14.

36 Annema W, Tietge UJ. Role of hepatic lipase and endothelial lipase in highdensity lipoprotein-mediated reverse cholesterol transport. Curr Atheroscler Rep 2011;13:257-65

37 McInnes IB, Thompson L, Giles JT, et al. Effect of interleukin- 6 receptor blockade on surrogates of vascular risk in rheumatoid arthritis: MEASURE, a randomised, placebocontrolled study. Ann Rheum Dis 2015;74:694-702.

38 Yue X, Wu M, Jiang $\mathrm{H}$, et al. Endothelial lipase is upregulated by interleukin-6 partly via the $\mathrm{p} 38 \mathrm{MAPK}$ and $\mathrm{p} 65 \mathrm{NF}-\mathrm{\kappa B}$ signaling pathways. Mol Med Rep 2016:14:1979-85. 